\title{
Associação entre proteínas do plasma seminal, motilidade e viabilidade espermática em coelhos submetidos a doping genético
}

\author{
[Association among seminal plasma proteins, sperm motility and sperm viability \\ in rabbits submitted to gene doping] \\ G. Urtiaga, V.F. Campos", T.F. Collares, P.M.M. Leon, J.C. Deschamps, \\ F.K. Seixas, T. Collares \\ Universidade Federal de Pelotas - UFPel - Pelotas, RS
}

\begin{abstract}
RESUMO
Neste trabalho foi estudada a correlação entre o perfil proteico do plasma seminal e a motilidade e viabilidade espermática em coelhos submetidos ao tratamento com vetores de expressão contendo o gene da eritropoetina (EPO) e com EPO recombinante humana. Foram identificadas, em coelhos submetidos ao tratamento com vetor de DNA contendo o gene da EPO, duas bandas proteicas associadas a alterações na motilidade espermática $-48 \mathrm{kDa}$ à baixa motilidade $(\mathrm{P}<0,05)$ e $18 \mathrm{kDa}$ à alta motilidade $(\mathrm{P}<0,05)$ - e esse fator foi associado a maior viabilidade espermática $(\mathrm{P}<0,05)$. Em coelhos submetidos ao tratamento com EPO recombinante, um fator proteico, $63 \mathrm{kDa}$, associou-se à alta motilidade espermática $(\mathrm{P}<0,05)$, enquanto dois, 26 e 40kDa, foram associados à alta viabilidade espermática $(\mathrm{P}<0,05)$. Esses resultados sugerem que o doping genético pode ocasionar mudanças no perfil proteico do plasma seminal, provocando alterações na motilidade e viabilidade espermática.
\end{abstract}

Palavras-chave: reprodução, eritropoetina, proteína, sêmen, transfecção

\begin{abstract}
In this study the correlation between seminal plasma protein profile and the sperm motility and sperm viability in rabbits submitted to treatment with an expression vector containing EPO gene and with human recombinant EPO was evaluated. In rabbits submitted to treatment with EPO expression vector, two protein bands were associated to sperm motility $-48 \mathrm{kDa}$ associated to low motility $(P<0.05)$ and $18 k D a$ to high motility $(P<0.05)$ - and this protein band was also associated to high sperm viability $(P<0.05)$. In rabbits submitted to treatment with human recombinant EPO, a protein factor, $63 \mathrm{kDa}$, was associated to high sperm motility $(P<0.05)$ while two protein factors, 26 and $40 \mathrm{kDa}$, were associated to high sperm viability $(P<0.05)$. These results suggest that gene doping leads to changes in rabbit seminal plasma protein, altering sperm motility and sperm viability.
\end{abstract}

Keywords: reproduction, erythropoietin, protein, sperm, transfection

\section{INTRODUÇÃO}

O doping genético caracteriza-se pela utilização não-terapêutica de genes, elementos genéticos e/ou células que potencializem a performance atlética (Wells, 2009). Seu uso é proibido em competições esportivas, porém ainda não foi desenvolvido um mecanismo eficiente de detecção dessa prática. Dentre os genes candidatos à prática de doping genético, estão o do hormônio do crescimento, os reguladores da miostatina e o da eritropoetina (EPO) (Haisma e De, 2006; Baoutina et al., 2010; Thevis et al., 2011).

A EPO é um hormônio glicoproteico com importante função na regulação da eritropoese em resposta às alterações nos níveis de oxigênio

Recebido em 3 de junho de 2011

Aceito em 3 de setembro de 2012

*Autor para correspondência (corresponding author)

E-mail: fariascampos@gmail.com 
circulante e, embora seu uso seja ilegal, sua forma recombinante é muito utilizada em competições esportivas devido ao seu efeito de incremento na capacidade aeróbica, inclusive em corridas de cavalo, caracterizando o doping (Jelkmann, 2003; Toutain, 2010). O uso da EPO recombinante em corridas de cavalo caracterizase como uma problemática pelos efeitos indesejados à saúde animal (Bailly-Chouriberry et al., 2010; Yu et al., 2010).

Os reflexos reprodutivos do uso da EPO ainda não foram descritos in vivo. Estudos in vitro, a partir do cultivo de células, demonstraram que seu uso estimula a esteroidogênese, provocando aumento da produção de testosterona em células de Leydig, sugerindo que a administração de EPO in vivo afeta a espermatogênese (Mioni et al., 1992; Yamazaki et al., 2004).

O plasma seminal é uma mistura de fluidos secretados pelo testículo, epidídimo e glândulas sexuais acessórias, e contém diferentes componentes, tais como açúcares, lipídeos, proteínas e íons. O plasma seminal está envolvido na manutenção das funções espermáticas e na fertilização (Wojtczak et al., 2005; Kareskoski e Katila, 2008).

O papel do plasma seminal e suas proteínas na maturidade espermática têm sido amplamente estudados, apresentando resultados contrastantes nas diferentes espécies animais. Em humanos, touros e carneiros, o plasma seminal auxilia a motilidade espermática através do incremento da viabilidade e integridade de membrana das células (Pickett et al., 1975; Baas et al., 1983). Vasconcelos et al. (2009) demonstraram que o perfil proteico do plasma seminal de cavalos muda ao longo da maturação sexual e sugeriram que proteínas inibidoras de proteases presentes no plasma podem atuar como marcadores de qualidade seminal em equinos. Por outro lado, em coelhos, estudos demonstraram que alterações na concentração do plasma seminal podem potencializar manutenção espermática, obtendo-se um incremento nos parâmetros de motilidade espermática a partir da adição de outros componentes ou aumento da diluição do sêmen (Castellini et al., 2000).

Este trabalho teve o objetivo de avaliar a correlação entre o perfil das proteínas do plasma seminal e a motilidade espermática em coelhos submetidos a doping genético e convencional.

\section{MATERIAL E MÉTODOS}

Foram utilizados 12 coelhos adultos da raça Nova Zelândia Albino, com pesos entre 4,5 e $5 \mathrm{~kg}$, mantidos no Biotério Central da Universidade Federal de Pelotas (UFPel), em gaiolas unitárias, sob condições controladas de alimentação, temperatura e iluminação. O estudo foi aprovado pelo Comitê de Ética em Experimentação Animal da UFPel (Registro $n^{\mathbf{o}}$ 200935). Os animais foram distribuídos em três grupos de quatro animais (Tab. 1). O grupo I recebeu três injeções subcutâneas semanais de $25 \mathrm{UI} / \mathrm{kg}$ de rHuEPO comercial diluída em solução salina ao longo de cinco semanas. O grupo II recebeu duas injeções intramusculares no dia 0 do estudo de uma dose de $200 \mu \mathrm{g}$ do vetor contendo o gene da eritropoetina (pTarget/EPO) cada uma. O grupo III (controle) recebeu duas injeções intramusculares contendo a mesma quantidade de vetor, porém com o gene da eritropoetina ausente (pTarget). A rHuEPO comercial (grupo I) utilizada nos testes foi Eprex (Issy-les-Moulinaux, France) e os vetores (grupo II e III) previamente construídos (Collares, 2010; Collares et al., 2012).

Tabela 1. Grupos do estudo e seus correspondentes dados de tratamento submetido, via de inoculação, dosagem inoculada e número de animais

\begin{tabular}{cccc}
\hline Grupo* $^{*}$ & Tratamento & Via & Dosagem \\
\hline I & rHuEPO & Subcutânea & $25 \mathrm{UI} / \mathrm{Kg}$ \\
II & pTarget/EPO & Intramuscular & $2 \times 200 \mu \mathrm{g}$ \\
III & Controle & Intramuscular & $2 \times 200 \mu \mathrm{g}$ \\
\hline
\end{tabular}

$*_{\mathrm{n}}=4$ animais/grupo.

Um ejaculado por macho por semana foi coletado usando vagina artificial ao longo do estudo. Do sêmen coletado, foram realizadas avaliações de motilidade e viabilidade espermática. A motilidade espermática foi medida em amostras diluídas 1:10 em Ringer com lactato de sódio (BASA Pharmaceutical Industry, BRA) a $37^{\circ} \mathrm{C}$ por dois avaliadores, de 
forma independente, em microscópio com contraste de fase e aumento de 200x. A viabilidade espermática foi obtida por meio do kit Live/Dead Sperm Viability kit (Invitrogen), de acordo com o protocolo sugerido pelo fabricante.

Uma alíquota de sêmem coletado de cada macho ao longo do estudo, uma vez por semana, foi centrifugada a $3000 \mathrm{~g}$ por $10 \mathrm{~min}$, a fim de separar os espermatozoides e a porção plasmática do sêmen. A SDS-PAGE foi realizada como previamente descrita por Campos et al. (2010). Os géis foram corados à temperatura ambiente com Coomassie Brilliant Blue (Sigma Chemical CO. - St. Louis, EUA) em over-night. Os géis foram analisados com o software TotalLab TL 100 v. 2006 (Nonlinear Dynamics - Newcastle, Inglaterra).

Amostras de sangue $(1 \mathrm{ml})$ foram coletadas uma vez por semana ao longo das cinco semanas do estudo, obtidas da artéria auricular. As amostras foram enviadas para o Laboratório de Análises Clínicas Ary Costa (Pelotas, RS), o qual utilizou o método automatizado ABX Micros 60 e a microscopia óptica para realização de hemograma completo.

Para o número de eritrócitos, usou-se análise de variância seguida de teste Tukey para a comparação de médias. Os dados de motilidade espermática foram categorizados em menor que
$70 \%$ e igual ou maior que $70 \%$, enquanto os de viabilidade espermática em menor que $80 \%$ e igual ou maior que $80 \%$. Com isso associou-se a motilidade e/ou viabilidade espermática à presença ou ausência de cada banda proteica no plasma seminal através do teste de MannWhitney.

\section{RESULTADOS}

Diferenças nas características sanguíneas foram observadas nos diferentes grupos e diferentes tempos (Collares, 2010). O grupo I (rHuEPO) apresentou incremento no número de eritrócitos ao longo do trabalho $(\mathrm{P}<0,05)$. O grupo II (pTarget/EPO) demonstrou incremento no número de eritrócitos durante a primeira semana do estudo $(\mathrm{P}<0,05)$. O grupo III (controle) manteve o número de hemácias constantes ao longo do estudo.

A análise in silico dos géis de poliacrilamida resultantes da SDS-PAGE demonstraram a presença de dezenas de bandas, muitas vezes sobrepostas e de difícil separação padronizada. A partir de tais análises, foram, portanto, eleitas dez bandas proteicas mais claramente identificadas. Os fatores encontrados apresentaram pesos moleculares entre 18 e 190kDa (Tab. 2; Fig. 1). Apenas duas bandas, 33 e $95 \mathrm{kDa}$, estavam presentes em todas a amostras coletadas.

Tabela 2. Bandas selecionadas pelo estudo para análise, seus respectivos pesos moleculares e frequência de ocorrência nas amostras totais e por grupo, em coelhos

\begin{tabular}{cccccc}
\hline Banda & Peso & $\begin{array}{c}\text { Frequência } \\
\text { total }\end{array}$ & $\begin{array}{c}\text { Frequência } \\
\text { grupo I }\end{array}$ & $\begin{array}{c}\text { Frequência } \\
\text { grupo II }\end{array}$ & $\begin{array}{c}\text { Frequência } \\
\text { grupo III }\end{array}$ \\
\hline 1 & $190 \mathrm{kDa}$ & 0,9166 & 0,9523 & 0,8695 & 0,9375 \\
2 & $95 \mathrm{kDa}$ & 1 & 1 & 1 & 1 \\
3 & $63 \mathrm{kDa}$ & 0,55 & 0,5714 & 0,4347 & 0,6875 \\
4 & $59 \mathrm{kDa}$ & 0,7833 & 0,8571 & 0,7391 & 0,75 \\
5 & $48 \mathrm{kDa}$ & 0,45 & 0,3333 & 0,4782 & 0,5625 \\
6 & $40 \mathrm{kDa}$ & 0,1166 & 0,0952 & 0,1304 & 0,125 \\
7 & $33 \mathrm{kDa}$ & 1 & 1 & 1 & 1 \\
8 & $29 \mathrm{kDa}$ & 0,05 & 0 & 0,0434 & 0,125 \\
9 & $26 \mathrm{kDa}$ & 0,1833 & 0,1904 & 0,1739 & 0,1875 \\
10 & $18 \mathrm{kDa}$ & 0,9 & 0,8571 & 0,8695 & 1 \\
\hline
\end{tabular}



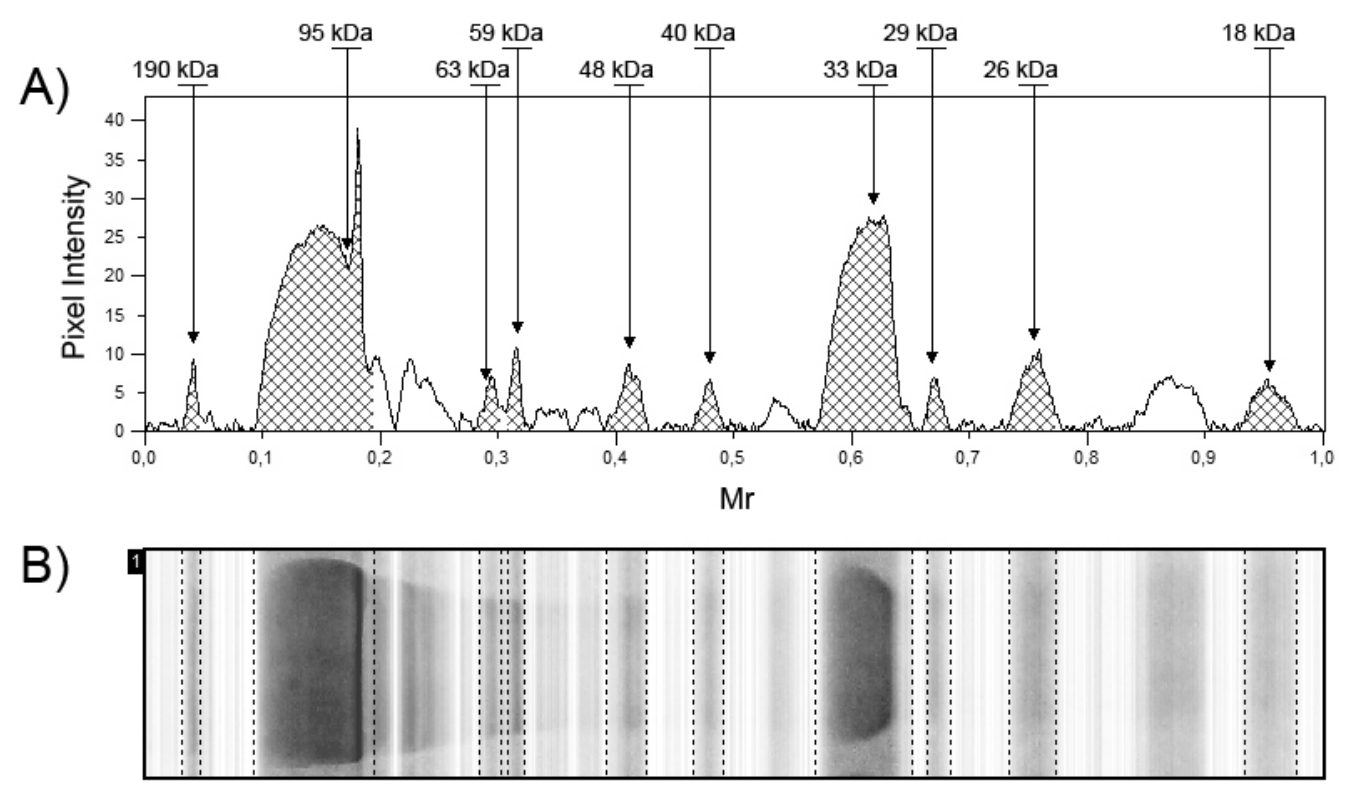

Figura 1. (A) intensidade de pixel de cada banda presente no plasma seminal dos coelhos. As setas apontam o peso molecular de cada banda. Mr: mobilidade relativa.kDa: kiloDalton. (B) SDS-PAGE de uma amostra contendo todas as bandas proteicas selecionadas para análise no trabalho.

Nos coelhos submetidos ao tratamento com EPO recombinante, grupo I, apenas o fator proteico de $63 \mathrm{kDa}$ demonstrou relação direta com a motilidade espermática. A presença dessa banda proteica está relacionada à motilidade espermática acima de $70 \% \quad(\mathrm{P}<0,05)$. Nos coelhos submetidos ao doping genético, grupo II, a presença do fator proteico de $48 \mathrm{kDa}$ está correlacionada à motilidade espermática abaixo de $70 \%(\mathrm{P}<0,05)$. Contrariamente, a presença da banda proteica de $18 \mathrm{kDa}$ correlacionou-se positivamente à motilidade, demonstrando que sua presença relaciona-se à alta motilidade $(\mathrm{P}<0,05)$.

Quanto à viabilidade espermática, as análises demonstraram que a presença dos fatores proteicos de 26 e $40 \mathrm{kDa}$, de forma isolada, relaciona-se à maior viabilidade espermática (acima de 80\%; $\mathrm{P}<0,05$ ) em coelhos submetidos ao doping convencional, grupo I. A presença do fator proteico de $18 \mathrm{kDa}$ relacionou-se, também, positivamente à maior viabilidade espermática em coelhos do grupo II $(\mathrm{P}<0,05)$, submetidos ao doping genético.

Nos coelhos do grupo controle não se observou relação entre a motilidade ou viabilidade espermática e a presença dos fatores proteicos analisados.

\section{DISCUSSÃO}

Este estudo demonstrou incremento no número de eritrócitos em coelhos submetidos ao tratamento com eritropoetina recombinante, confirmando a função regulatória dessa proteína na eritropoese (Collares, 2010). Demonstrou também o incremento no número das células vermelhas nos coelhos submetidos a doping com o gene da eritropoetina, confirmando resultados obtidos em estudos anteriores (Rizzuto et al., 1999; Fattori et al., 2005). Diferentemente de Rizzuto et al. (1999), este trabalho não submeteu os animais a diferentes formas de transfecção do plasmídio ao tecido, limitando-se à utilização de injeção intramuscular sem posterior eletroporação, que é um método de transfecção que utiliza um pequeno choque elétrico no tecido para aumentar a transfecção de DNA para a o tecido alvo. Provavelmente a eletroestimulação incrementaria os resultados (Rizzuto et al., 1999).

O padrão proteico do plasma seminal de coelhos encontrado neste estudo difere daquele descrito por Lavon (1972). Há carência de trabalhos nesse sentido, e novos trabalhos, buscando a caracterização proteica do plasma seminal de coelhos, seriam interessantes, em função da importância desse modelo mamífero. 
Mioni et al. (1992) demonstraram que a administração de EPO recombinante estimula o processo de esteroidogênese em células de leydig de ratos cultivadas in vitro, despertando a hipótese de que a EPO pode acarretar alterações na espermatogênese, visto que tais células são reguladoras da espermatogênese.

Posteriormente, estudos in vivo demonstraram que a administração de EPO recombinante em humanos provoca incremento na produção de testosterona (Foresta et al., 1994). Souza et al. (2010) relacionaram o aumento nos níveis de secreção andrógena com alterações na expressão de algumas proteínas do plasma seminal em carneiros. Yamazaki et al. (2004) identificaram a presença de receptores proteicos específicos para EPO na membrana de células de Leydig. Gacci et al. (2010) sugeriram que alterações nas concentrações de testosterona provocam modificações na motilidade espermática. Ergun et al. (2007) demonstraram que o incremento na produção de testosterona está relacionado à diminuição na motilidade espermática em humanos. Além disso, a associação entre testosterona, motilidade e alterações no plasma seminal foi demonstrada em coelhos (Yousef, 2005; Yousef et al., 2005). Assim, as proteínas podem ter sido alteradas pelo doping genético por EPO.

O presente trabalho é pioneiro na verificação de características reprodutivas em animais submetidos ao doping convencional administração de rHuEPO - e doping genético administração de vetores contendo o gene da eritropoetina. Sugere que fatores proteicos distintos correlacionam-se diferentemente com motilidade e viabilidade espermática, dependendo do tipo de tratamento ao qual o animal foi submetido. Outros estudos já demonstraram a influência de fatores proteicos na motilidade espermática (Muller e Kirchner, 1978). Segundo Campos et al. (2010), existe associação entre a presença do fator proteico de $38 \mathrm{kDa}$ e baixa motilidade em peixes (Rhamdia quelen). Yue et al. (2009) demonstraram que proteínas com 78, 49, 44, 25, 21 e $15 \mathrm{kDa}$ estavam associadas à baixa motilidade em ovinos. Outros estudos ainda demonstram a influência de diferentes fatores proteicos na motilidade espermática de caprinos (Dungdung e Majumder, 2003), aves (Mohan et al., 1995), humanos (Iwamoto e Gagnon, 1988) e bovinos (Baas et al., 1983).

Em relação à viabilidade espermática (integridade de membrana), outros estudos anteriores já identificaram um fator proteico de $26 \mathrm{kDa}$ associado à integridade de membrana plasmática superior a $55 \%$ em espermatozoides suínos pós-descongelamento (Bianchi et al., 2008). Bancos de dados in silico apontam que um grupo proteico de $26 \mathrm{kDa}$, chamado de Sialoproteínas, tem por função a inibição da aglutinação das cabeças dos espermatozoides. Strzezek (2002) apontou que a deficiência desse grupo pode comprometer o potencial espermático de fertilização. Yue et al. (2009) também identificaram proteínas de 72, 37 e $18 \mathrm{kDa}$ associadas à alta viabilidade espermática, enquanto um fator de $15 \mathrm{kDa}$ foi associado à baixa viabilidade espermática em ovinos. Além disso, Killian et al. (1993) correlacionaram o fator proteico de $26 \mathrm{kDa}$ à alta fertilidade em touros. Alvarez e Storey (1995) demonstraram que a Albumina presente no plasma seminal está envolvida tanto com a proteção da membrana plasmática quanto com a manutenção da motilidade, uma vez que essa proteína absorve o peróxido de lipídio, protegendo as células.

Embora vários estudos tenham encontrado associação entre proteínas do plasma seminal e características reprodutivas, ainda pouco se conhece sobre a maneira como essas proteínas agem sobre os espermatozoides. Estudos anteriores têm fornecido evidências de que agem por adsorção à membrana das células espermáticas (Desnoyers e Manjunath, 1992; Manjunath e Therien, 2002; Asadpour et al., 2007). Em bovinos, as proteínas BSP A1/A2 e BSP $30 \mathrm{kDa}$ são adsorvidas na membrana espermática e induzem o influxo de colesterol da membrana mediando a capacitação espermática (Manjunath e Therien, 2002). Desse modo, podemos hipotetizar que os fatores proteicos encontrados em associação no presente estudo devem agir por adsorção à membrana das células. Contudo, mais estudos são necessários para elucidar esse mecanismo. 


\section{CONCLUSÃO}

A administração de rHuEPO e vetores contendo o gene da eritropoetina provocam alterações nos perfis proteicos do plasma seminal e, consequentemente, alterações na motilidade e viabilidade espermática em coelhos.

\section{AGRADECIMENTOS}

T.F. Collares é aluna do Programa de PósGraduação em Biotecnologia da UFPel e bolsista CAPES. F.K. Seixas, J.C. Deschamps e T. Collares são Bolsistas de Produtividade do CNPq.

\section{REFERÊNCIAS} incorporation of fatty acids into and peroxidative loss of fatty acids from phospholipids of human spermatozoa. Mol. Reprod. Dev., v.42, p.334-346, 1995.

ASADPOUR, R.; ALAVI-SHOUSHTARI, S.M.; REZAII, S.A.; ANSARI, M.H. SDS-polyacrylamide gel electrophoresis of buffalo bulls seminal plasma proteins and their relation with semen freezability. Anim. Reprod. Sci., v.102, p.308-313, 2007.

BAAS, J.W.; MOLAN, P.C.; SHANNON, P. Factors in seminal plasma of bulls that affect the viability and motility of spermatozoa. J. Reprod. Fertil., v.68, p.275-280, 1983.

BAILLY-CHOURIBERRY， L.; NOGUIER， F.; MANCHON, L. et al. Blood cells RNA biomarkers as a first long-term detection strategy for EPO abuse in horseracing. Drug Test. Anal., v.2, p.339-345, 2010.

BAOUTINA, A.; COLDHAM, T.; BAINS, G.S.; EMSLIE, K.R. Gene doping detection: evaluation of approach for direct detection of gene transfer using erythropoietin as a model system. Gene Ther., v.17, p.1022-1032, 2010.

BIANCHI, I.; COLLARES, T.; CAMPOS, V.F. et al. Seminal plasma factor associated to post-thawing swine sperm membrane integrity. Arq. Bras. Med. Vet. Zootec., v.60, p.384-388, 2008.

CAMPOS, V.F.; SEIXAS, F.K.; KAEFER, C. et al. Association between the presence of a $38 \mathrm{kDa}$ factor in the seminal plasma anda inhibition of sperm motility in jundiá fish Rhamdia quelen. Cienc. Anim. Bras., v.11, p.402-409, 2010.

CASTELLINI, C.; LATTAIOLI, P.; MORONI, M.; MINELLI, A. Effect of seminal plasma on the characteristics and fertility of rabbit spermatozoa. Anim. Reprod. Sci., v.63, p.275-282, 2000.
ALVAREZ, J.G.; STOREY, B.T. Differential

COLlARES, T.F. Efeito da administração de eritropoetina $e$ de vetores recombinantes em parâmetros reprodutivos de coelhos. 2010. (51f.) Dissertação (Mestrado em Biotecnologia) - Centro de Biotecnologia, Unversidade Federal de Pelotas, Pelotas, RS.

COLLARES, T.F.; CAMPOS, V.F.; URTIAGA, G. et al. Erythropoietin non-viral gene therapy does not affect motility, viability, morphology or concentration of rabbit sperm. Animal, in press, 2012

DESNOYERS, L.; MANJUNATH, P. Major proteins of bovine seminal plasma exhibit novel interactions with phospholipids. J. Biol. Reprod., v.267, p.1014910155, 1992.

DUNGDUNG, S.R.; MAJUMDER, G.C. Isolation and identification of a novel motility-inhibiting factor from goat cauda sperm plasma membrane. Cell Mol. Biol., v.49, p.413-420, 2003.

ERGUN, A.; KOSE, S.K.; AYDOS, K. et al. Correlation of seminal parameters with serum lipid profile and sex hormones. Arch. Androl., v.53, p.2123, 2007.

FATTORI, E.; CAPPELLETTI, M.; ZAMPAGLIONE, I. et al. Gene electro-transfer of an improved erythropoietin plasmid in mice and nonhuman primates. J. Gene Med., v.7, p.228-236, 2005.

FORESTA, C.; MIONI, R.; BORDON, P. et al. Erythropoietin stimulates testosterone production in man. J. Clin. Endocrinol. Metabol., v.78, p.753-756, 1994.

GACCI, M.; CORONA, G.; APOLONE, G. et al. Influence of serum testosterone on urinary continence and sexual activity in patients undergoing radical prostatectomy for clinically localized prostate cancer. Prostate Cancer Prostatic Dis., v.13, p.168-172, 2010.

HAISMA, H.J.; HON, O. Gene doping. Int. J. Sports Med., v.27, p.257-266, 2006.

IWAMOTO, T.; GAGNON, C. A human seminal plasma protein blocks the motility of human spermatozoa. J. Urol., v.140, p.1045-1048, 1988.

JELKMANN, W. Erythropoietin. J. Endocrinol. Invest. v.26, p.832-837, 2003.

KARESKOSKI, M.; KATILA, T. Components of stallion seminal plasma and the effects of seminal plasma on sperm longevity. Anim. Reprod. Sci., v.107, p.249-256, 2008.

KILLIAN, G.J.; CHAPMAN, D.A.; ROGOWSKI, L.A. Fertility-associated proteins in Holstein bull seminal plasma. Biol. Reprod., v.49, p.1202-1207, 1993. 
LAVON, U. Characterization of boar, bull, ram and rabbit seminal plasma proteins by gel disc electrophoresis and isoelectric focusing on polyacrylamide. J. Reprod. Fertil., v.31, p.29-37, 1972.

MANJUNATH, P.; THERIEN, I. Role of seminal plasma phospholipid-binding proteins in sperm membrane lipid modification that occurs during capacitation. J. Reprod. Immunol., v.53, p.109-119, 2002.

MIONI, R.; GOTTARDELLO, F.; BORDON, P. et al. Evidence for specific binding and stimulatory effects of recombinant human erythropoietin on isolated adult rat Leydig cells. Acta Endocrinol., v.127, p.459-465, 1992.

MOHAN, J.; SAINI, M.; JOSHI, P. Isolation of a spermatozoa motility inhibiting factor from chicken seminal plasma with antibacterial property. Biochim. Biophys. Acta, v.1245, p.407-413, 1995.

MULLER, B.; KIRCHNER, C. Influence of seminal plasma proteins on motility of rabbit spermatozoa. $J$. Reprod. Fertil., v.54, p.167-172, 1978.

PICKETT, B.W.; SULLIVAN, J.J.; BYERS, W.W. et al. Effect of centrifugation and seminal plasma on motility and fertility of stallion and bull spermatozoa. Fertil. Steril., v.26, p.167-174, 1975.

RIZZUTO, G.; CAPPELLETTI, M.; MAIONE, D. et al. Efficient and regulated erythropoietin production by naked DNA injection and muscle electroporation. Proc. Natl. Acad. Sci. U.S.A, v.96, p.6417-6422, 1999.

SOUZA, C.E.; ARAUJO, A.A.; OLIVEIRA, J.T. et al. Reproductive development of Santa Ines rams during the first year of life: body and testis growth, testosterone concentrations, sperm parameters, age at puberty and seminal plasma proteins. Reprod. Domest. Anim., v.45, p.644-653, 2010.

STRZEZEK, J. Secretory activity of boar seminal vesicle glands. Reprod. Biol., v.2, p.243-266, 2002.

THEVIS, M.; GEYER, H.; THOMAS, A.; SCHANZER, W. Trafficking of drug candidates relevant for sports drug testing: Detection of nonapproved therapeutics categorized as anabolic and gene doping agents in products distributed via the Internet. Drug Test. Anal., v.3, p.331-336, 2011.
TOUTAIN, P.L. Veterinary medicines and competition animals: the question of medication versus doping control. Handb. Exp. Pharmacol., v.1, p.315-339, 2010.

VASCONCELOS, A.B.; SANTOS, A.M.; OLIVEIRA, J.S. et al. Purification and partial characterization of proteinase inhibitors of equine seminal plasma. Reprod. Biol., v.9, p.151-160, 2009.

WELLS, D.J. Gene doping: possibilities and practicalities. Med. Sport Sci., v.54, p.166-175, 2009.

WOJTCZAK, M.; DIETRICH, G.J.; CIERESZKO, A. Transferrin and antiproteases are major proteins of common carp seminal plasma. Fish Shellfish Immunol., v.19, p.387-391, 2005.

YAMAZAKI, T.; KANZAKI, M.; KAMIDONO, S.; FUJISAWA, M. Effect of erythropoietin on Leydig cell is associated with the activation of Stat5 pathway. Mol. Cell Endocrinol., v.213, p.193-198, 2004.

YU, N.H.; HO, E.N.; WAN, T.S.; WONG, A.S. Doping control analysis of recombinant human erythropoietin, darbepoetin alfa and methoxy polyethylene glycol-epoetin beta in equine plasma by nano-liquid chromatography-tandem mass spectrometry. Anal. Bioanal. Chem., v.396, p.25132521, 2010.

YUE, W.; SHI, L.; BAI, Z. et al. Sodium dodecyl sulfate (SDS)-polyacrylamide gel electrophoresis of ram seminal plasma proteins and their correlation with semen characteristics. Anim. Reprod. Sci., v.116, p.386-391, 2009.

YOUSEF, M.I.; EL-MORSY, A.M.; HASSAN, M.S. Aluminium-induced deterioration in reproductive performance and seminal plasma biochemistry of male rabbits: protective role of ascorbic acid. Toxicology, v. 215 , p. $97-107,2005$.

YOUSEF, M.I. Reproductive performance, blood testosterone, lipid peroxidation and seminal plasma biochemistry of rabbits as affected by feeding Acacia saligna under subtropical conditions. Food Chem. Toxicol., v.43, p.333-339, 2005. 